\title{
Tailored polyaniline/barium strontium titanate/ expanded graphite multiphase composite for efficient radar absorption
}

\author{
Pradeep Sambyal, ${ }^{a}$ Avanish Pratap Singh, ${ }^{a}$ Meenakshi Verma, ${ }^{b}$ M. Farukh, ${ }^{a}$ \\ Bhanu Pratap Singh ${ }^{c}$ and S. K. Dhawan*a
}

\begin{abstract}
The present paper reports the synthesis of a high-performance microwave absorbing material using a simple, cost-effective and scalable method by encapsulating barium strontium titanate (BST) and expanded graphite (EG) in a polyaniline (PANI) matrix. One of the formulations (higher content of BST) shows shielding effectiveness due to absorption of more than $50 \mathrm{~dB}$ (>99.9999\% attenuation) with minimum reflection loss $(\leq 1 \mathrm{~dB})$ in the $\mathrm{Ku}$-band $(12.4-18 \mathrm{GHz})$ frequency range. Another formulation (higher content of EG) shows a total shielding effectiveness of more than $81 \mathrm{~dB}$ with a reflection loss of $10 \mathrm{~dB}$. In order to probe the relationship between the observed shielding response and the electromagnetic attributes, dielectric and permeability parameters have been calculated from the measured scattering parameters $\left(S_{11}, S_{22}, S_{12}, S_{21}\right)$ using the Nicolson-Ross-Weir algorithm. The synthesised formulations were characterized thoroughly using XRD, FTIR, TGA, UV, Raman spectroscopy, SEM and HRTEM.
\end{abstract}

Received 8th November 2013 Accepted 20th January 2014

DOI: $10.1039 / c 3 r a 46479 b$

www.rsc.org/advances the total EMI shielding effectiveness $\left(\mathrm{SE}_{\mathrm{T}}\right)$ is the sum of the contributions from absorption $\left(\mathrm{SE}_{\mathrm{A}}\right)$, reflection $\left(\mathrm{SE}_{\mathrm{R}}\right)$ and transmission or multiple reflections $\left(\mathrm{SE}_{\mathrm{M}}\right)$. However, when $\mathrm{SE}_{\mathrm{T}}$ $>10 \mathrm{~dB}, \mathrm{SE}_{\mathrm{M}}$ can be ignored. ${ }^{4,5}$ Furthermore, the effective absorbance $\left(A_{\text {eff }}\right)$ can be described as $A_{\text {eff }}=(1-R-T) /(1-R)$ with respect to the power of the effectively incident EM wave inside the shielding material, where $R$ and $T$ are the reflectance and transmittance coefficients. For a material, the skin depth $(\delta)$ is the distance up to which the intensity of the EM wave decreases to $1 / e$ of its original strength. $\delta$ is related to the angular frequency, relative permeability and total conductivity, $\sigma_{\mathrm{T}}=\left(\sigma_{\mathrm{dc}}+\sigma_{\mathrm{ac}}\right)$. According to EM theory, for electrically thick samples $(t>\delta)$, the frequency $(\omega)$ dependence of far field losses can be expressed in terms of the total conductivity $\left(\sigma_{\mathrm{T}}\right)$, real permeability $\left(\mu^{\prime}\right)$, skin depth $(\delta)$ and thickness $(t)$ of the shield material as: $\mathrm{SE}_{\mathrm{R}}(\mathrm{dB})=10 \log \left\{\sigma_{\mathrm{ac}} / 16 \omega \varepsilon_{0} \mu^{\prime}\right\}$, and $\mathrm{SE}_{\mathrm{A}}(\mathrm{dB})=20\{t / \delta\} \log e=20 d \sqrt{\mu \omega \sigma_{\mathrm{ac}} / 2} \log e=8.68\{t / \delta\} . \sigma_{\mathrm{ac}}$ and $\delta$ can be related to the imaginary permittivity $\left(\varepsilon^{\prime \prime}\right)$ and real permeability $\mu^{\prime}$ by $\sigma_{\mathrm{ac}}=\omega \varepsilon_{0} \varepsilon^{\prime \prime}$ and $\delta=\sqrt{2 / \sigma \omega \mu^{\prime}}$, which gives the absorption loss as: $\mathrm{SE}_{\mathrm{A}}(\mathrm{dB})=8.68 t \sqrt{\sigma \omega \mu^{\prime} / 2}$. In the microwave range, the contribution of $\mathrm{SE}_{\mathrm{A}}$ becomes more, as compared to $\mathrm{SE}_{\mathrm{R}}$. This may be attributed to the shallow skin depth and high conductivity $\left(\sigma_{\mathrm{ac}}\right)$ values at such high frequencies. ${ }^{4}$

Thus the EMI shielding effectiveness (SE) of a composite material mainly depends on the filler's intrinsic conductivity, dielectric constant and wt\% ratio. ${ }^{4}$ Generally, the EMI SE is governed by the dielectric properties, magnetic permeability, thickness and frequency. ${ }^{6}$ Therefore, in the recent past, 
composites that are electrically conducting (metals, conducting polymers, carbon black, graphite, carbon fibers, carbon nanotubes, graphene) and dielectric $\left(\mathrm{TiO}_{2}, \mathrm{SiO}_{2}, \mathrm{ZnO}\right.$, fly ash, barium titanate, strontium titanate, barium strontium titanate) have been employed for EMI shielding. ${ }^{7-10}$ Among all of them, electrically conducting polymer composites have gained popularity recently because of their properties such as wide range of conductivity, light weight, resistance to corrosion, flexibility and processability. ${ }^{2}$ Polyaniline (PANI) is unique among the family of conjugated polymers, as its doping level can be readily controlled through an acid doping/base dedoping process. PANI is an electrically conducting polymer with many features that can be exploited in various applications. The preparation of particles with controlled core-shell morphology is one of the ways to improve the processibility of this polymer and to obtain electrically conducting composites. ${ }^{11}$ The conductivity of PANI composites can be further improved using fillers such as expanded graphite (EG), multiwalled carbon nanotubes and metals. In any composite, excellent electrical conductivity along with a high dielectric constant are two important parameters for the suppression of reflection or enhancement of microwave absorption. In view of the above, PANI composites have been synthesized with EG, as EG sheets occupy the interface of macromolecules and nanoscale objects. Besides the high electrical conductivity, the large surface area and low ${ }^{\mathbf{1 2}}$ wt $\%$ of EG make it a unique candidate to provide remarkable EMI shielding ${ }^{\mathbf{1 3}}$ in light weight polymer composites. Graphitic materials have exhibited potential in improving the electrical conductivity of polymers. ${ }^{14}$ Furthermore, the dielectric needs of PANI-EG composites can be fulfilled by barium strontium titnate (BST) as it is shows a high dielectric constant. For the first time, we demonstrate its use for EMI shielding applications. Prior to this, BST has been extensively investigated for various applications such as piezoelectric sensors, dynamic random access memories, microwave phase shifters, uncooled infrared detectors, microwave devices and gas sensors due to its excellent compositional and structural modifiability. Therefore, EG (as conducting filler) and BST (as high permittivity filler) are incorporated in appropriate amounts within a PANI (conducting polymer) matrix to mitigate EM pollution.

In the present work, BST nanoparticles and EG have been incorporated into PANI by in situ polymerization. The addition of BST results in an enhancement in the microwave absorption. The paper reports the electrical and microwave absorption properties of PANI/BST/EG (PBE) composites prepared by the in situ emulsion polymerization of aniline with BST and EG in the presence of dodecyl benzene sulfonic acid (DBSA). The different compositions have been prepared by taking different concentrations of BST and EG in PANI, and are referred to as PBE composites. It is noteworthy that the SE strongly depends on the dielectric loss and weight fraction of BST in the polyaniline matrix. We expect that our composite could act as an ideal microwave absorber with minimum reflectance and may potentially be used in EMI shielding applications and as a radar absorbing material.

\section{B. Experimental}

\section{Materials}

Aniline (Rankem Limited, India), ethylene glycol, (Himedia, India) ammonium persulfate (APS, Rankem Limited, India), citric acid (Merck, India), titanium isoproxide (Himedia, India), nitric acid (Merck, India), strontium carbonate (Sigma Aldrich, India), barium carbonate (Merck, India), DBSA (Merck, India) were used in the present study. The aniline monomer was purified by distillation under reduced pressure before use. Natural graphite powder (Loba Chemie, India; purity 99.5\%, particle size $50 \mu \mathrm{m}$ ) was used to synthesize EG. Aqueous solutions were prepared using double deionized water with a specific resistivity of $10^{6} \Omega \mathrm{cm}$. The other chemicals were of reagent grade and used as received.

\section{Synthesis of the PANI/BST/EG composite}

The PBE composites were prepared using BST, EG, aniline, DBSA and ammonium persulfate. Prior to PBE composite synthesis, BST and EG were prepared as follows. For the synthesis of BST nanoparticles, a modified polymerized citrate precursor method was used. ${ }^{15}$ In a typical reaction, $0.1 \mathrm{~mol}$ of titanium tetrabutoxide was first dissolved into $2 \mathrm{~mol}$ of ethylene glycol. The resulting solution was stirred until it became transparent and yellow. Subsequently, citric acid (CA) was directly added to this solution. A small amount of water (about $0.5 \mathrm{~mL}$ water per gram of $\mathrm{CA}$ ) was added into the solution to help dissolve the citric acid. A white precipitate was observed at first but immediately dissolved after they were stirred for $30 \mathrm{~min}$ on a magnetic stirrer, and the solution became clear. $0.07 \mathrm{~mol}$ and $0.03 \mathrm{~mol}$ of $\mathrm{BaCO}_{3}$ and $\mathrm{SrCO}_{3}$ powders respectively were added to this solution. Three drops of nitric acid $\left(\mathrm{HNO}_{3}, 65-68 \%\right)$ for every $100 \mathrm{~mL}$ of mixture were added to the sample to catalyze the dissolving of the carbonates and esterification between citric acid and ethylene glycol. The mixture was stirred continuously until all of the powder was dissolved, leading to a transparent and pale brown solution. This solution was immersed in a basin with hot silicon oil $\left(50-60{ }^{\circ} \mathrm{C}\right)$ and stirred for $12 \mathrm{~h}$. Then it was placed into an oven and dried at $140{ }^{\circ} \mathrm{C}$ to promote polymerization and remove excess solvent. With continued heating, the solution became a viscous resin with a change in color from pale brown to deep brown. No visible precipitation was observed during the polymerization. The resulting resin was charred at $300{ }^{\circ} \mathrm{C}$ for 2 $\mathrm{h}$ in an electric furnace, resulting in a porous and black solid mass. The charred precursor was lightly ground into a powder with a mortar and pestle. Heat treatment of this precursor at $800-900{ }^{\circ} \mathrm{C}$ for $8 \mathrm{~h}$ in a muffle furnace resulted in white BST powders.

The synthesis of EG nanosheets was carried out using chemical exfoliation by simply controlling the oxidation and exfoliation procedure. ${ }^{\mathbf{1 6 , 1 7}}$ Natural graphite was blended with a mixture of concentrated sulfuric acid and concentrated nitric acid ( $3: 1$ weight ratio) by stirring at room temperature and kept static for $24 \mathrm{~h}$. It was observed that the suspension gradually evolved brown fumes from the solution, during which the 
bulk graphite powder was transformed into EG nanosheets. The product was obtained after filtration, washed with distilled water until reaching pH 7 and dried for $6 \mathrm{~h}$ at $90{ }^{\circ} \mathrm{C}$.

The resulting EG and BST nanoparticles were ultra-homogenized in a $0.2 \mathrm{M}$ aqueous solution of DBSA with an appropriate amount of $0.1 \mathrm{M}$ distilled aniline monomer for $2.5 \mathrm{~h}$ to form a homogeneous dispersion. The above solution thus polymerized at $-3{ }^{\circ} \mathrm{C}$ through chemical oxidation polymerization using ammonium persulfate $(0.1 \mathrm{M})$ as a oxidant. The resulting solution was treated with an equal amount of isopropyl alcohol to dissolve the oligomers which are formed during the reaction. The resulting precipitates were filtered out and washed with water twice. The resulting composite was dried at $65-70{ }^{\circ} \mathrm{C}$. Polymer composites with different weight ratios of BST to aniline were prepared.

PANI (electrically conducting matrix or host) can offer advantages such as good electrical and dielectric properties which may collectively contribute towards high microwave absorption efficiency. Several compositions with different PANI : BST : EG weight ratios, i.e. $1: 0.1: 1,1: 0.2: 1$ and $1: 0.1: 2$ were prepared and designated as PBE111, PBE121 and PBE112, respectively.

\section{Materials characterization}

The morphology of PANI-DBSA and its composites were examined using scanning electron microscopy (SEM, Zeiss EVO MA-10). The SEM samples were prepared by dispersing the powder in isopropanol using ultra-sonication and placing small drops of the suspension on silicon wafers and sputter-coating with gold before analysis. High-resolution transmission electron microscopy (HRTEM) was performed using a Technai G20stain $300 \mathrm{kV}$ instrument. The semi-crystalline nature of PANIDBSA and the PBE composites was confirmed by X-ray diffraction (XRD) studies carried out on a D8 Advance XRD instrument (Bruker) using $\mathrm{Cu} \mathrm{K} \alpha$ radiation $(\lambda=1.54 \AA)$ in the scattering range $(2 \theta)$ of $10^{\circ}-70^{\circ}$ with a scan rate of $0.02^{\circ} \mathrm{sec}^{-1}$ and a slit width of $0.1 \mathrm{~mm}$. The electrical conductivity of the PBE composites was measured by a standard four-probe technique using a Keithley programmable current source (model 6221) and nanovoltmeter (model 2182A) attached to a digital temperature controller (Lakeshore 331) at room temperature. For the electrical conductivity measurements, rectangular pellets $\left(13 \times 7 \mathrm{~mm}^{2}\right)$ were prepared from the powder samples using a rectangular die and a hydraulic press at a compression

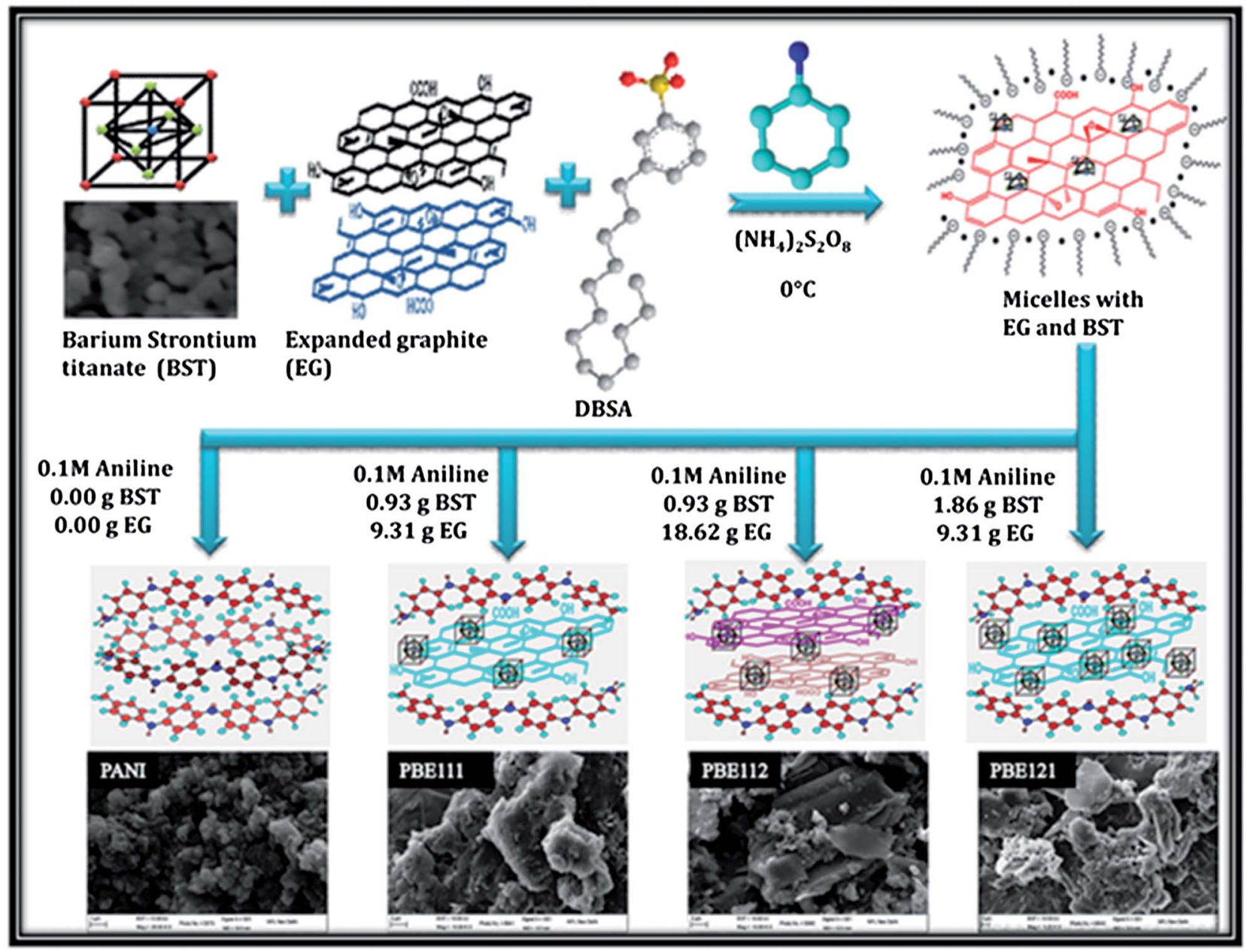

Fig. 1 Schematic representation of BST nanoparticle-decorated EG sheets filled in PANI by the in situ polymerization of aniline using APS as the oxidant in the presence of DBSA. 
pressure of 5 tons. Four ohmic contacts were made on each end of the pellet using silver epoxy paste. A thermogravimetric analyzer (Mettler Toledo TGA/SDTA 851e) was used to measure the thermal stability of the material under an inert atmosphere (flowing $\mathrm{N}_{2}$ gas) in the temperature range of $25-900{ }^{\circ} \mathrm{C}$. FTIR spectra were recorded on a Nicolet 5700 instrument in the transmission mode in the wave number range of $400-4000 \mathrm{~cm}^{-1}$. Spectroscopic-grade $\mathrm{KBr}$ pellets were used for collecting the spectra with a resolution of $4 \mathrm{~cm}^{-1}$, performing 32 scans. UV-vis absorption studies were carried out on a Shimadzu 1601 spectrophotometer. Raman analysis was carried out using a Renishaw inVia Reflex spectrometer (UK) with an excitation source of 514.5 $\mathrm{nm}$. The resolution of the instrument was less than $1.0 \mathrm{~cm}^{-1}$. EMI shielding and dielectric measurements were carried out using an Agilent E8362B vector network analyzer in the 12.4-18 $\mathrm{GHz}$ (Ku-band) microwave range. Powder samples were compressed in the form of rectangular pellets $(\sim 2.5 \mathrm{~mm}$ thick $)$ and inserted in a copper sample holder connected between the wave-guide flanges of the network analyzer.

\section{Results and discussion}

The PBE composites were prepared by in situ emulsion polymerization using aniline/BST/EG as the dispersed phase and DI water as the continuous phase. DBSA molecules possesses surfactant characteristics with a hydrophilic $\mathrm{SO}_{3} \mathrm{H}$ head and hydrophobic tail, and easily forms micelles in aqueous solution. As shown in Fig. 1, BST and EG were dispersed in DBSA aqueous solution before polymerization. As a result, micelles containing BST and EG form in the reaction. These micelles have a core-shell structure, where BST and EG particles reside in the core of the micelles due to their hydrophobic nature, while DBSA is regarded as the "shell" due to its hydrophilic $\mathrm{SO}_{3} \mathrm{H}$ group. Further, aniline monomer was emulsified with DBSA to form an aniline/DBSA salt via an acid/base reaction. The aniline/DBSA micelles act as a soft template with the formation of an oil in a water-type emulsion. The addition of an oxidant (APS) to the solution initiates the polymerization at the micelle-water interface. Thus, aniline is oxidised and forms anilinium radical cations, which subsequently combine with another unit to form a neutral dimer. Further oxidation of this dimer leads to the formation of a trimer, tetramer and finally the polymer composites incorporated with BST and EG particles form. A schematic representation of the incorporation of BST and EG into the polyaniline matrix is shown in Fig. 1. The above scheme suggests that BST and EG embedded in PANI leads to the formation of the PBE composite which has better electrical and dielectric properties.

\section{Surface morphology}

The morphologies of BST, EG, PANI and the PANI composites were investigated using SEM. Fig. 2(a) illustrates the BST

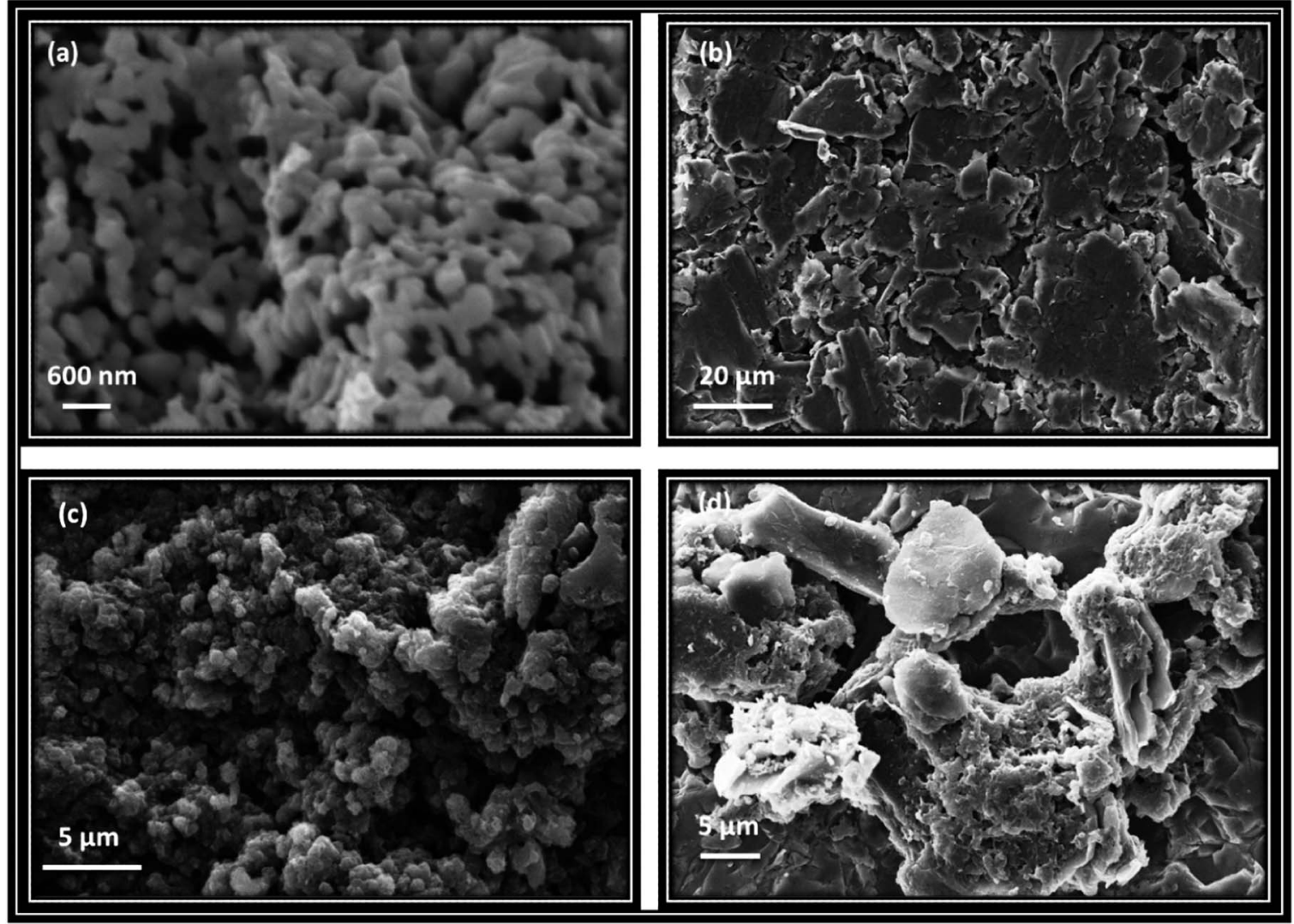

Fig. 2 SEM images of (a) BST nanoparticles synthesized by the citrate precursor method, (b) expanded graphite, (c) PANI-DBSA and (d) the PBE121 composite. 
nanoparticles synthesized by the citric precursor method. In general, the chemically derived BST nanoparticles agglomerate, similar to previous observations. ${ }^{18}$ The EG consists of folded and wrinkled sheets that are randomly aggregated, and their sizes are up to a few micrometers, as seen in Fig. 2(b). Fig. 2(c) shows the SEM image of DBSA-doped PANI. The resulting PANI powder was homogeneous and formed agglomerates with differently sized rock shapes. The SEM image of the PBE121 composite revealed the presence of BST particles and EG sheets. The BST particles appear as bright dots due to their nanostructure, and EG appears as sheets (Fig. 2(d)). The presence of conduction and the dielectric nanoparticles in the PANI matrix are helpful for sufficient impedance matching, which is necessary for enhancing the absorption of the EM wave.

TEM was carried out to determine the distribution of EG sheets and BST in the PANI matrix. Fig. 3(a) shows the TEM image of the EG sheets, while the inset shows the enlarged view of the lattice fringes. The average inter-planer spacing in the EG sheets is found to be $0.34 \mathrm{~nm}$, which is in excellent agreement with the $d$ spacing found by XRD. The TEM image of the PANI composite reveals the presence of EG sheets as darker areas and BST particles as the darkest spots, indicated using arrows in Fig. 3(b). It has been observed that the incorporation of BST nanoparticles along with EG in the PANI matrix greatly reduces the agglomeration tendency of BST particles due to the coating of DBSA-doped PANI. Therefore, the average size of the BST particles increases from $43 \mathrm{~nm}$ to $120 \mathrm{~nm}$.

\section{XRD}

Fig. 4(a) shows the XRD patterns of PANI (0.2 M DBSA-doped PANI), EG, BST and their composites with different weight ratios of aniline to BST i.e. PBE111, PBE112 and PBE121. PANI shows the characteristics peaks at $2 \theta=18.8$ and 25.35 , corresponding to the (100) and (110) crystal planes respectively. ${ }^{19}$ The XRD spectrum of BST shows highly crystalline perovskite cubic symmetry with reflection peaks at $2 \theta$ values of $22.32^{\circ}, 31.95^{\circ}$, $39.05^{\circ}, 45.49^{\circ}, 51.15^{\circ}$ and $56.39^{\circ}$ corresponding to the (100), (110), (111), (200), (210) and (211) sets of diffraction planes, respectively, which match with JCPDS card no. 34-0411. ${ }^{20}$ The XRD pattern of EG shows a sharp peak at $2 \theta=26.3^{\circ}$, which corresponds to the (002) crystal plane. ${ }^{16}$ The nanocomposites show the characteristic peaks of BST and EG and their intensity increases monotonically with the increase in their content. It is seen that all of the detectable peaks of EG and BST are well retained and no other peak is observed, indicating that no chemical reaction occurred between PANI, BST and EG. These observations indicate that there is no change in the morphology of BST, although it was coated by PANI particles. The presence of BST is observed in the XRD studies because X-rays, having high energy, can penetrate the coated BST particles and reveal the presence of BST. ${ }^{21}$ However, the characteristic peak of PANI at $2 \theta=25.35$, due to the (110) plane, is masked by the intense peak of the expanded graphite and appears as a faint shoulder.

The crystallite size of the BST particles can be calculated by using the Debye-Scherrer formula:

$$
D=k \lambda / \beta \cos \theta
$$

where $D$ is the crystallite size for individual peaks, $\lambda$ is the X-ray wavelength, $K$ is the shape factor, $\theta$ is the half angle in degrees, and $B$ is the full width at half-maximum of the sample. The value of $k$ is often assigned a value of 0.89 , which depends on several factors, including the Miller index of the reflecting plane and the shape of the crystal. The (111) reflection of the observed $\mathrm{X}$-ray data was chosen for calculating the grain size of the BSTs. The average size of the BST particles was calculated using the above equation and estimated as $43 \mathrm{~nm}$.

\section{Thermogravimetric analysis}

Fig. 4(b) shows the thermal stability of PANI, EG, BST and their composites with different weight ratios of BST to PANI, i.e. PBE111, PBE112, PBE121. Pure EG showed excellent thermal stability up to $700{ }^{\circ} \mathrm{C}$ and only $\sim 2 \%$ weight loss is observed. BST, with exceptionally high thermal stability, shows no weight

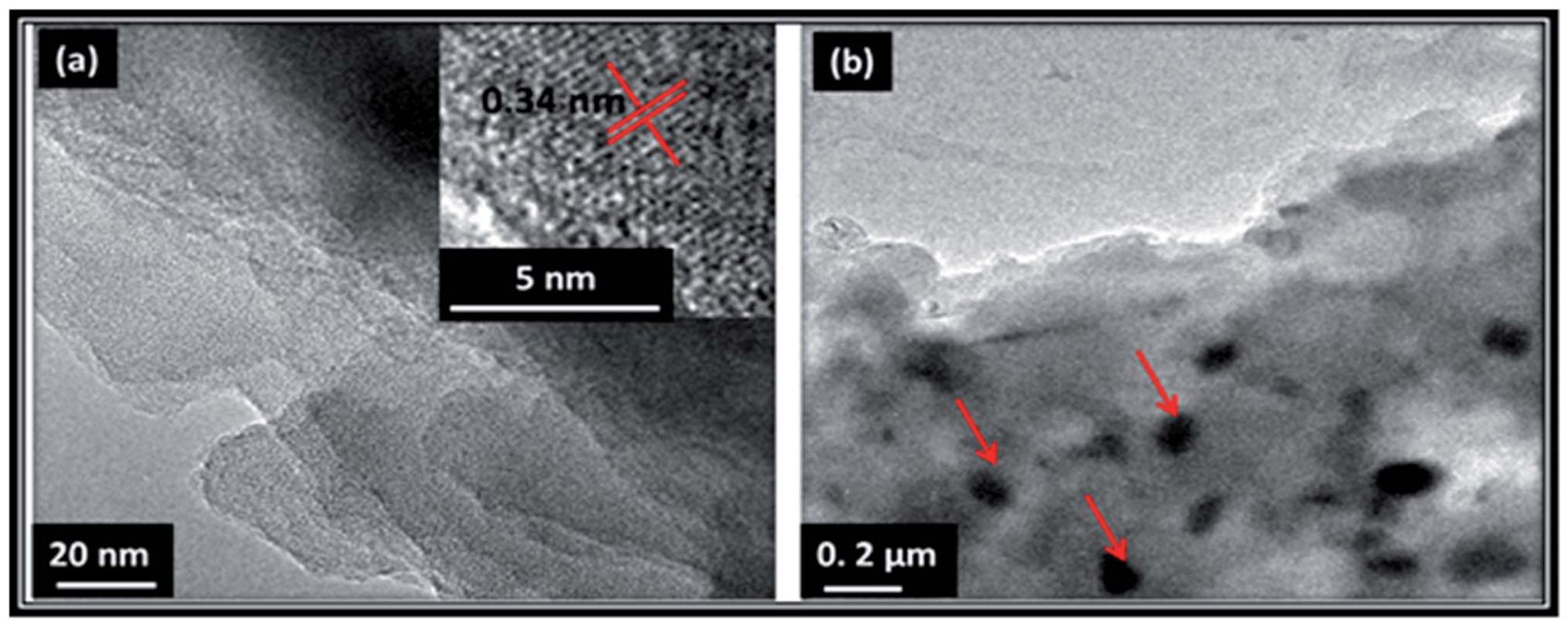

Fig. 3 TEM image of the (a) EG sheets (the inset shows the high resolution TEM lattice fringes of the EG sheets) and (b) PANI composite (PBE121). 

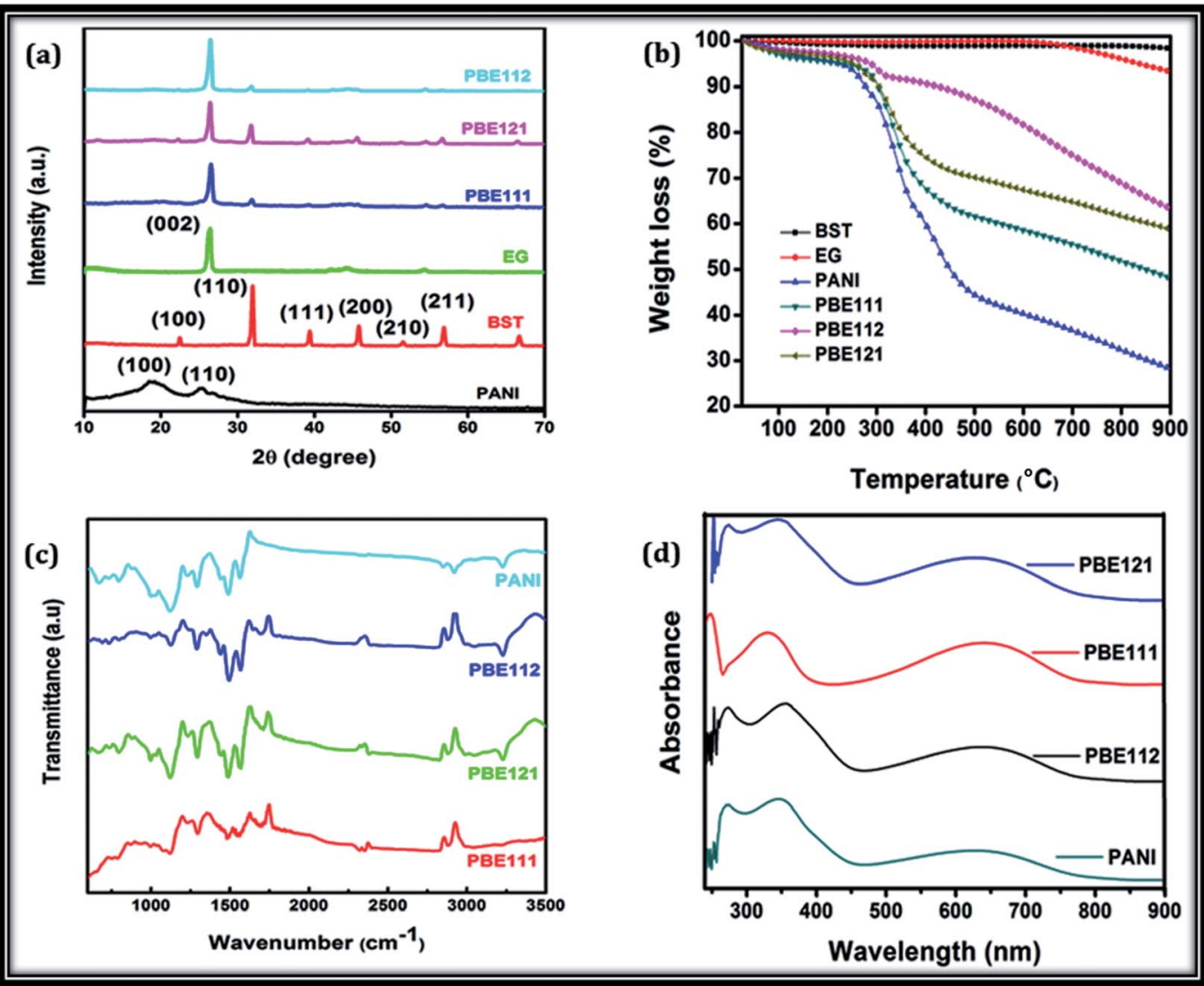

Fig. 4 (a) XRD patterns of BST, PANI-DBSA, PBE111, PBE121 and PBE112, (b) thermogravimetric (TG) plots of the PANI/BST composites with different BST contents in a $\mathrm{N}_{2}$ atmosphere, (c) comparison of the FTIR spectra of BST, PANI-DBSA, PBE111, PBE121 and PBE112, (d) UV-visible spectra of the PANI/BST/EG composites.

loss over the entire temperature range. PANI and its composites show two-stage degradation behavior. The first stage corresponding to $5-10 \%$ weight loss from room temperature to $200{ }^{\circ} \mathrm{C}$ is due to the expulsion of moisture and residual solvent in the composite samples. The major weight loss at above $341{ }^{\circ} \mathrm{C}$ is attributed to the degradation of PANI. ${ }^{19}$ The relative thermal stability of the composites was compared by comparing the $T_{5}$ (temperature at which weight loss is $5 \%$ ), $T_{10}$ (temperature for $10 \%$ weight loss), $T_{15}$ (temperature at which $15 \%$ weight loss was observed) and $T_{\max }$ (temperature at the maximum weight loss), as shown in Table 1. All these temperatures showed an increase upon the incorporation of EG and BST.

Table 1 The relative thermal stability of the composites was compared by comparing the $T_{5}$ (temperature at which weight loss is $5 \%), T_{10}$ (temperature for $10 \%$ weight loss), $T_{15}$ (temperature at which $15 \%$ weight loss was observed) and $T_{\max }$ (temperature at the maximum weight loss)

\begin{tabular}{lcccc}
\hline Sample designation & $T_{5}$ & $T_{10}$ & $T_{15}$ & $T_{\max }$ \\
\hline PANI & 225 & 277 & 307 & 341 \\
PBE111 & 231 & 303 & 321 & 335 \\
PBE112 & 287 & 425 & 545 & 301 \\
PBE121 & 255 & 303 & 327 & 329
\end{tabular}

With the addition of BST and EG, the $15 \%$ weight loss temperature increases from $307^{\circ} \mathrm{C}$ (PANI) to $321{ }^{\circ} \mathrm{C}, 545^{\circ} \mathrm{C}$ and $327{ }^{\circ} \mathrm{C}$ for composites PBE111, PBE112 and PBE121, respectively. The increase in the thermal stability of the composite samples over PANI is due to the enhancement of interactions between the PANI matrix, EG and BST nanoparticles, which hinder the segmental mobility of the polymer chains. ${ }^{22}$ The slow degradation of the PBE112 sample is accredited to the large amount of EG present in the sample. The TGA data shows that the PBE112 sample with a high loading of EG has the highest thermal stability over PANI and the other composites.

\section{FTIR}

Fig.4(c) shows the FTIR spectra of PANI and its nanocomposites. The band corresponding to the out-of-plane bending vibration of $\mathrm{C}-\mathrm{H}$ in the $p$-disubstituted benzene ring appears at $790 \mathrm{~cm}^{-1} \cdot{ }^{19}$ The bands at $1560 \mathrm{~cm}^{-1}$ and $1486 \mathrm{~cm}^{-1}$ are assigned to the $\mathrm{C}=\mathrm{C}$ stretching deformation of the quinoid and benzenoid rings, respectively. The bands at $1288 \mathrm{~cm}^{-1}$ and $1122 \mathrm{~cm}^{-1}$ are attributed to the stretching mode of the $\mathrm{C}-\mathrm{N}$ bond in secondary aromatic amines and the vibration mode of the $\mathrm{N}=\mathrm{Q}=\mathrm{N}$ ring, respectively. The broad band observed around $2915 \mathrm{~cm}^{-1}$ is due to the aromatic $\mathrm{C}-\mathrm{H}$ stretching vibrations and the band at $3222 \mathrm{~cm}^{-1}$ is due to the $\mathrm{N}-\mathrm{H}$ 
stretching of aromatic amines. ${ }^{23-25}$ These results are in good agreement with the previous spectroscopic characterization of PANI. ${ }^{\mathbf{2 6} 27}$ However, in the case of the nanocomposites, a slight shifting and broadening of the characteristic bands was observed which indicates the presence of physical interactions between PANI, BST and EG. The absence of any new vibration bands suggests van der Waalstype interactions without any chemical origin.

\section{UV-visible spectroscopy}

The UV absorption spectra of the different samples of polyaniline and its composites with EG and BST were recorded for determining the band gap. The main peaks and associated transition energies are shown in Fig. 4(d) and Table 2, respectively. The emeraldine base form of polyaniline in $N$-methyl pyrrolidinone (NMP) shows two characteristic bands at $326(\pi-$ $\pi^{*}$ transition) and $630 \mathrm{~nm}$ (quinine-diimine), while the characteristic bands of the conducting form of polyaniline (doped with DBSA) are observed at 272, 345 and $627 \mathrm{~nm}$. This blue shift, as well as the appearance of a new band (345 nm) on doping may be associated with the dopant-induced change in the supermolecular structure and formation of localized states (polarons/bipolarons). Therefore, the 345 and $627 \mathrm{~nm}$ bands are associated with polaronic transitions, i.e. polaron $-\pi^{*}$ and $\pi-$ polaron transitions, respectively. In the case of the polyaniline composite (PBE112), two changes are observed. Firstly, a red shift is observed for the band from 627 to $639 \mathrm{~nm}$, which may be due to the extension of the conjugation length caused by the weakened steric hindrance between the sulfonate and imine radical ions. Secondly, a red shift in the $\pi-\pi^{*}$ transition (345 to $355 \mathrm{~nm}$ ) is observed. This may be attributed to the changes in the chain morphology and the increase in effective conjugation or the increase in EG content. This is in accordance with the increase in conductivity with increasing EG content in the composite. The optical band gap of the polymer was obtained from the relation given below and the results are presented in Table 1:

$$
\alpha h v=A \sqrt{h v-E_{\mathrm{g}}}
$$

where $\alpha$ is the absorbance, $A$ is a constant, $h v$ is the photon energy and $E_{\mathrm{g}}$ is the optical band gap. The band gaps calculated by using the above equation varies from 1.62 to $1.65 \mathrm{eV}$ and 2.95 to $3.31 \mathrm{eV}$ for the polaronic and $\pi-\pi^{*}$ transitions of the benzenoid ring, respectively.

Table 2 DC electrical conductivity, band gap and shielding effectiveness of the PANI/BST/EG composites

\begin{tabular}{|c|c|c|c|c|c|c|}
\hline \multirow{2}{*}{$\begin{array}{l}\text { Sample } \\
\text { name }\end{array}$} & \multirow{2}{*}{$\begin{array}{l}\text { Conductivity } \\
\left(\mathrm{S} \mathrm{cm}^{-1}\right)\end{array}$} & \multicolumn{2}{|c|}{$\begin{array}{l}\text { Band gap (ev) } \\
\left(\alpha h v=A\left(h v-E_{\mathrm{g}}\right)^{1 / 2}\right)\end{array}$} & \multicolumn{3}{|c|}{$\begin{array}{l}\text { Shielding } \\
\text { effectiveness (dB) }\end{array}$} \\
\hline & & $\pi-\pi^{*}$ & $\pi$-polaron & $\mathrm{SE}_{\mathrm{A}}$ & $\mathrm{SE}_{\mathrm{R}}$ & $\mathrm{SE}_{\mathrm{T}}$ \\
\hline PANI & 1.70 & 3.06 & 1.65 & 17.75 & 8.66 & 26.41 \\
\hline PBE111 & 65.56 & 3.31 & 1.65 & 59.49 & 11.67 & 71.16 \\
\hline PBE112 & 187.18 & 2.95 & 1.62 & 71.26 & 10.28 & 81.54 \\
\hline PBE121 & 36.66 & 3.00 & 1.62 & 53.32 & 0.96 & 54.28 \\
\hline
\end{tabular}

\section{Raman spectroscopy}

Raman spectroscopy is an important tool to identify the interactions or bonding between polymeric materials and fillers. ${ }^{28}$ Fig. 5 shows the Raman spectra of PANI, BST, EG and the PBE111 composite. The Raman spectrum of EG consists of three prominent characteristic peaks, namely the D band, G band, and $\mathrm{G}^{\prime}(2 \mathrm{D})$ band, and their peak positions are identified at 1352, 1582 and $2711 \mathrm{~cm}^{-1}$ respectively. The inset shows the Raman spectrum of pure BST, which reveals the characteristic bands of BST in the low frequency region i.e. 220 and $520 \mathrm{~cm}^{-1}$. The $220 \mathrm{~cm}^{-1}$ peak is attributed to the mixed $\mathrm{TO}_{2}-\mathrm{LO}_{1}$ phonon mode, and the $520 \mathrm{~cm}^{-1}$ peak corresponds to the $\mathrm{TO}_{4}$ phonon. ${ }^{29}$ Bands at 1186 and $1623 \mathrm{~cm}^{-1}$, assigned to $\mathrm{C}-\mathrm{H}$ and $\mathrm{C}-\mathrm{C}$ of the benzenoid units respectively, are observed in the PANI spectrum. ${ }^{30}$ The same bands were observed for the composites but with a slight blue shift, i.e. 1186 to $1189 \mathrm{~cm}^{-1}$ and 1623 to 1624 $\mathrm{cm}^{-1}$. Another band at $1345 \mathrm{~cm}^{-1}$ related to the $\mathrm{C}-\mathrm{N}^{+}$stretching modes of delocalized polaronic charge carriers, which is characteristic of the protonated imine form of polyaniline, was also observed. ${ }^{31}$ This band was shifted to $1355 \mathrm{~cm}^{-1}$ from $1345 \mathrm{~cm}^{-1}$ with the incorporation of BST and graphite. The slight shifting in the bands is evidence of the formation of interactions between BST, PANI and EG. The low frequency mode of BST was also observed in the composite sample due to the presence of BST.

\section{Dielectric and EMI shielding measurements}

The room temperature DC electrical conductivity of the composites is shown in Table 2 . EG sheets possess very good conductivity, ${ }^{9}$ therefore, the conductivity escalates sharply with a higher wt\% loading of EG i.e., from 1.70 (PANI) to $187.18 \mathrm{~S}$ $\mathrm{cm}^{-1}$ (PBE112). Most importantly, these samples show optimum values of conductivity, which is desired for exhibiting good microwave shielding responses. ${ }^{32}$ Hence, one would expect that these materials could be potentially used in

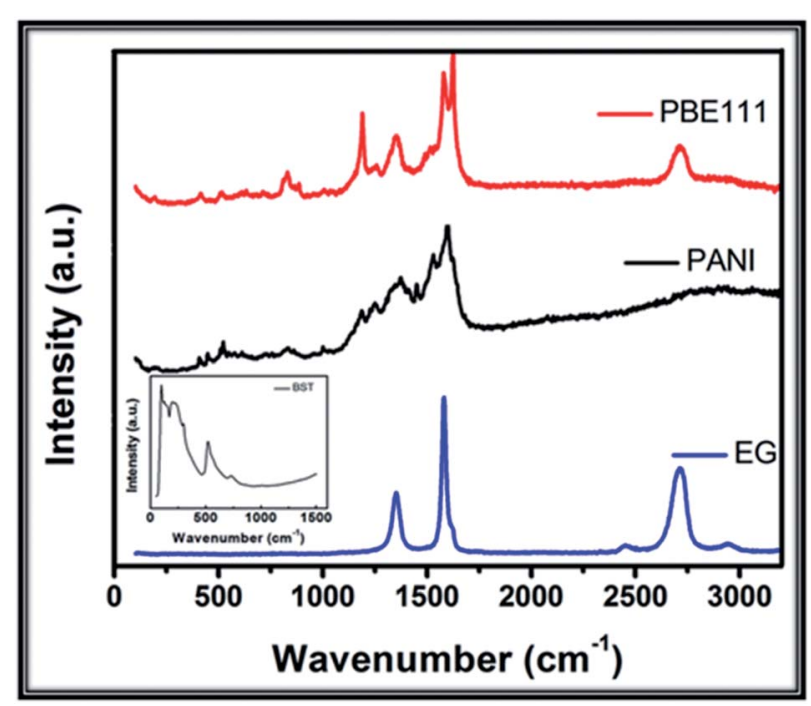

Fig. 5 Raman spectra of EG, PANI-DBSA and PBE111. The inset shows the Raman spectra of BST. 
applications such as in electrostatic charge dissipation (ESD) and in EMI shielding. Here, we have focused mainly on the EMI shielding applications, rather than its use in ESD. Highly conducting materials such as carbon nanotubes and exotic carbon materials have been primarily demonstrated for EMI shielding applications. ${ }^{33}$ Few attempts have been made to introduce EG along with the polymer for enhancing the EMI shielding effectiveness. ${ }^{34-36}$

The moderate electrical conductivity and complex permittivity are two governing parameters for the suppression of reflection or improvement of microwave absorption. Therefore, the incorporation of EG (as a conducting filler) and BST (as a high permittivity filler) in appropriate amounts within the PANI (conducting polymer) matrix is expected to improve the absorption of EM pollution. In view of the fact that our composite demonstrates optimum conductivity along with a high dielectric constant, it is highly desirable for its use in EMI shielding applications.

Fig. 6(a) shows the total SE, while Fig. 6(b) illustrates the $\mathrm{SE}_{\mathrm{A}}$ and $\mathrm{SE}_{\mathrm{R}}$ of PANI and its composites. $\mathrm{SE}$ values of $26,71,81$ and $54 \mathrm{~dB}$ are achieved for PANI, PBE111, PBE112 and PBE121,
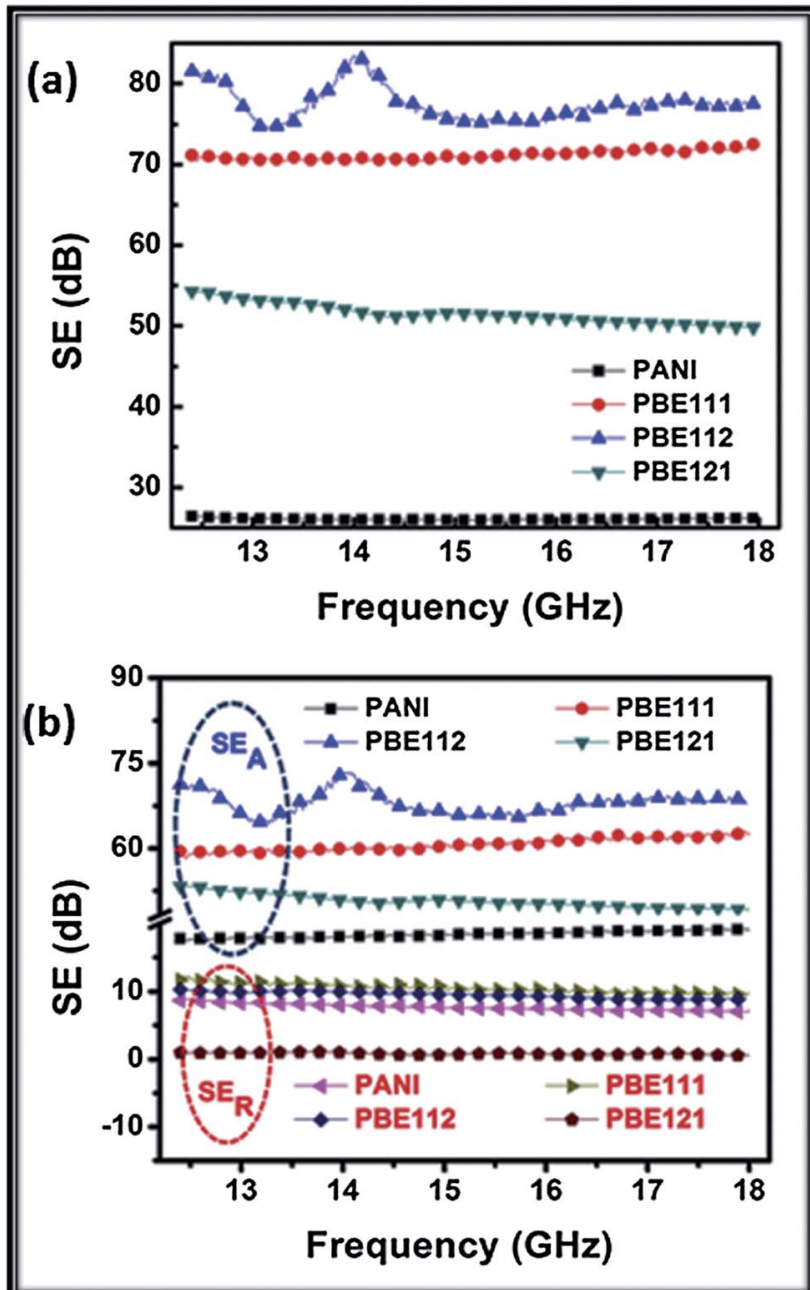

Fig. 6 (a) EMI SE, and (b) $\mathrm{SE}_{\mathrm{A}}$ and $\mathrm{SE}_{\mathrm{R}}$ of PANI and its composites as a function of frequency. respectively. Composite PBE121 gives a very interesting SE result, with $\mathrm{SE}_{\mathrm{A}}$ more than $50 \mathrm{~dB}$ and $\mathrm{SE}_{\mathrm{R}}$ less than $1 \mathrm{~dB}$ over the entire frequency range. From the detailed shielding analysis, it is observed that at $15.0 \mathrm{GHz}$, the $\mathrm{SE}_{\mathrm{A}}$ value increases with the increase in $\mathrm{EG}$ content, while the $\mathrm{SE}_{\mathrm{R}}$ has no significant change. Moreover, $\mathrm{SE}_{\mathrm{A}}$ and $\mathrm{SE}_{\mathrm{R}}$ both decrease with the increasing content of BST in the PANI-EG matrix. When the BST content reaches $9 \mathrm{wt} \%$ in the PANI-EG matrix, the $\mathrm{SE}_{\mathrm{R}}$ values obtained are less than $1 \mathrm{~dB}$, which is the minimum value of reflection achieved so far with retaining optimum microwave absorption. EMI shielding using highly conducting materials such as metals is governed by reflection rather than absorption. ${ }^{3}$ On the other hand, hybrid conducting composites provide EMI shielding predominantly due to absorption, owing to the presence of electric dipoles. ${ }^{32,37}$ Analogously, we observed a similar phenomenon for our PANI composites.

In order to probe the relationship between the observed shielding response and the electromagnetic attributes, dielectric and permeability parameters were calculated from the measured scattering parameters $\left(S_{11}, S_{22}, S_{12}, S_{21}\right)$ using the Nicolson-Ross-Weir algorithm. Fig. 7(a) shows that, for PANI composites PBE111, PBE112 and PBE121, the values of $\varepsilon^{\prime}$ are in the range of 257.98-174.21, 663.91-356.62, and 155.77-91.98, respectively, which are higher than for the pristine PANI (40.8433.13 ), in the frequency range of 12.4 to $18 \mathrm{GHz}$. Meanwhile, the value of $\varepsilon^{\prime \prime}$ for PBE111, PBE112 and PBE121 are in the range of 219.81-150.26, 342.01-288.48 and 93.29-64.35, respectively, which are also higher than PANI (22.95-22.11), as shown in Fig. 7(b). However, some small and weak fluctuations were found in the $\varepsilon^{\prime \prime}$ curves, which can be attributed to different relaxation processes. ${ }^{\mathbf{4} 38}$ It is observed that the samples with a higher wt\% loading of BST show higher $\varepsilon^{\prime}$ and $\varepsilon^{\prime \prime}$ values due to the dielectric nature of BST. It is proposed that more EG plates may enhance the conductivity and electric polarization because the permittivity $\left(\varepsilon^{\prime}\right)$ is a measure of the polarizability of a material, which induces dipolar and electric polarization in the presence of microwaves. In addition, $\varepsilon^{\prime}$ is also improved by polarization that occurs due to the localized defects such as polarons and bipolarons present in the PANI matrix, something which has been reported previously. ${ }^{\mathbf{1 0 , 3 9}}$

As shown in Fig. 7(c) for PANI composites PBE111, PBE112 and PBE121, the values of $\mu^{\prime}$ are in the range of $0.05-0.09,1.30-$ 0.89 and $0.20-0.20$, respectively, which are higher than for pristine PANI (0.12-0.12) in the Ku-band. Meanwhile, the value of $\mu^{\prime \prime}$ for PBE111, PBE112 and PBE121 are in the range of 0.28$0.25,0.56-0.63$ and $0.12-0.15$, respectively, which are almost equal to PANI (0.18-0.14), as shown in Fig. 7(d). The observed $\mu^{\prime \prime}$ is always greater than 0.3 over the entire frequency range, indicating that the permeability loss occurs in all frequency ranges. Generally, permeability and its loss are found to be higher in polymer ferrite composites, ${ }^{\mathbf{1 7}}$ but in non-ferromagnetic composites their values are very small and similar to the results observed for PBE composites. These results suggest that PANI composites have distinct dielectric loss properties.

According to EM theory, dielectric losses in the shield are the combined result of complex phenomena such as natural resonance, dipole relaxation, polarization of charge carriers and its 


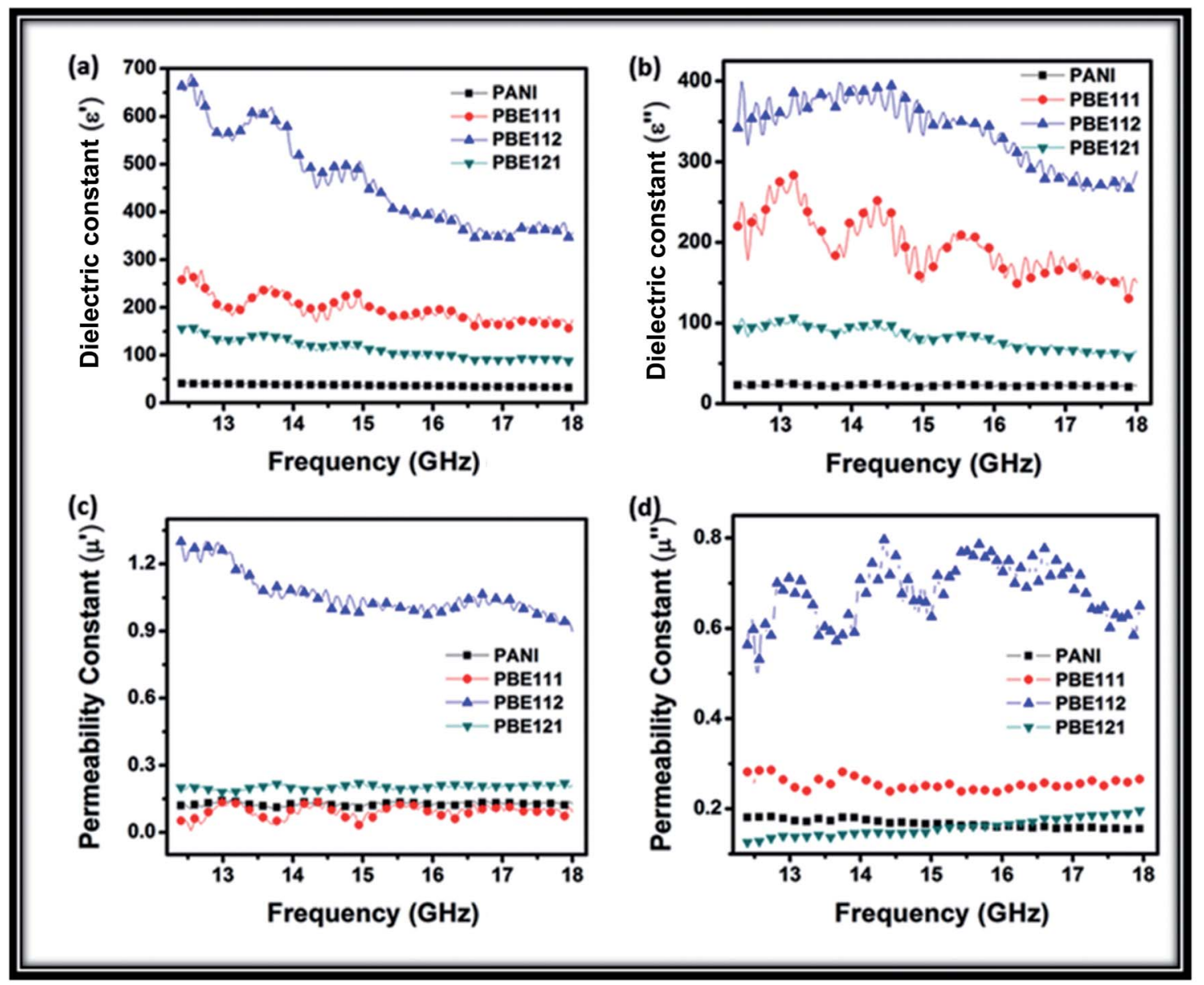

Fig. 7 Frequency dependence of the (a) real parts, (b) imaginary parts of the complex permittivity, (c) real parts and (d) imaginary parts of permeability of PANI composites.

relaxation, polarization of polarons and bipolarons in the polymer matrix and their relaxation, and certainly the unique structure of the shield. BST particles possess high dielectric constants, therefore these act as polarized centers inside the polymer matrix, which results in increased microwave absorption. Furthermore, highly conducting EG sheets also enhance the absorption properties. ${ }^{9,17,40}$ BST particles act as tiny dipoles which are polarized in the presence of an EM field, resulting in better microwave absorption. ${ }^{41}$ In the PANI composites, the existence of interfaces between BST particles and the EG layer, PANI and BST particles, and between PANI-PANI, are responsible for interfacial polarization which further contributes to dielectric losses. Interfacial polarization occurs in heterogeneous media due to the accumulation of charges at the interfaces and the formation of large dipoles. Some space charge is developed during activation by an EM wave on the surface of the PANI matrix due to the difference in the conductivity of PANI $\left(1.70 \mathrm{~S} \mathrm{~cm}^{-1}\right)$, BST $\left(\sim 10^{-12} \mathrm{~S} \mathrm{~cm}^{-1}\right)$ and EG $\left(1612.12 \mathrm{~S} \mathrm{~cm}^{-1}\right) .{ }^{9}$ This also contributes to interfacial polarization, and furthermore, the generation of space charge at the heterogeneous interface, leading to field distortion and accounting for the strong polarization in the system. The conductivity of the PANI composite is improved by EG and results in the increase of the dielectric loss, therefore $\varepsilon^{\prime \prime}$ increases with the higher wt\% loading of EG, as illustrated in Fig. 7(b). The natural resonances in the Ku-band can be attributed to the small size of BST. The anisotropy energy of the small-sized materials, ${ }^{42}$ especially in the nanoscale, would be higher due to the surface anisotropic field due to the small size effect. ${ }^{43}$ The combined effect of the above mentioned polarization effects and associated loss mechanisms are responsible for the high value of $\varepsilon^{\prime}$. The real permittivity $\left(\varepsilon^{\prime}\right)$ for any given composition decreases with frequency (Fig. 7(a)) because, as the frequency increases, the dipoles present in the polymer chain are not able to sustain their phase orientation with the electric vector of the incident wave. The higher anisotropy energy also contributes to the enhancement of the microwave absorption.

An SE value of around $30 \mathrm{~dB}$ is required for commercial EMI shielding applications, ${ }^{2}$ and an SE value of more than $80 \mathrm{~dB}$ is desired for high-tech defence applications. Therefore, among all of the PANI composites, PBE121 is potentially applied for making futuristic radar absorbers as well as mobile signal interference absorbers in daily life, while PBE112 can be used for high-tech EMI shielding applications. Interestingly, any desired value of SE, between two limits, can be achieved by scaling the loading level of BST and EG depending on the requirement of the specific sector. The newly synthesised composite gave the best SE due to absorption compared to 
Table 3 Electromagnetic shielding properties of different PANI-based composites

\begin{tabular}{|c|c|c|c|c|c|c|}
\hline \multirow[b]{2}{*}{ Filler } & \multirow[b]{2}{*}{ Filler wt\% } & \multirow{2}{*}{$\begin{array}{l}\text { Frequency } \\
\text { range }(\mathrm{GHz})\end{array}$} & \multicolumn{3}{|c|}{ Shielding effectiveness (dB) } & \multirow[b]{2}{*}{ Ref. } \\
\hline & & & $\mathrm{SE}_{\mathrm{A}}$ & $\mathrm{SE}_{\mathrm{R}}$ & $\mathrm{SE}_{\mathrm{T}}$ & \\
\hline $\mathrm{BaFe}_{12} \mathrm{O}_{19}$ & 75 & $12.4-18$ & 28.9 & 3.1 & 32 & Ohlan et al. ${ }^{46}$ \\
\hline MWCNT & 10 & $12.4-18$ & 88 & 10 & 98 & Gupta et al. ${ }^{47}$ \\
\hline $\mathrm{Fe}_{3} \mathrm{O}_{4}$ & 66 & $12.4-18$ & 9 & 2.5 & 11.5 & Singh et al. ${ }^{48}$ \\
\hline Carbon black & 30 & $2-18$ & - & - & 40 & Wu et al. ${ }^{49}$ \\
\hline $\mathrm{GF}\left(\mathrm{Fe}_{3} \mathrm{O}_{4} /\right.$ graphene $)$ & 66 & $12.4-18$ & 26 & 6.3 & 32.3 & Singh et al. ${ }^{48}$ \\
\hline $\mathrm{BaTiO}_{3}$ & 50 & $12.4-18$ & 65.6 & 5.9 & 71.5 & Saini et al. $^{38}$ \\
\hline $\mathrm{Sb}_{2} \mathrm{O}_{3}$ & 50 & $8.2-12.4$ & - & - & 21 & Faisal et $a .^{39}$ \\
\hline $\mathrm{BaFe}_{12} \mathrm{O}_{19} / \mathrm{TiO}_{2}$ & $25 / 50$ & $12.4-18$ & 58 & 1.8 & 59.8 & Ohlan et al. ${ }^{10}$ \\
\hline $\mathrm{CoFe}_{2} \mathrm{O}_{3}$ & 66 & $12.4-18$ & 21.5 & 3.5 & 26 & Gandhi et al. ${ }^{50}$ \\
\hline Graphene & 33 & $2.0-18$ & - & - & 34.2 & Bingqing et al. ${ }^{51}$ \\
\hline SWCNT & 25 & $2.0-18$ & & & 31.5 & Bingqing et al. ${ }^{51}$ \\
\hline EG-cobalt ferrite & 1 & $2.0-18$ & - & - & 19.3 & Chen et al. ${ }^{34}$ \\
\hline Fly ash & 75 & $12.4-18$ & 25 & 7 & 32 & Singh et $a .^{8}$ \\
\hline BST/EG & $9 / 45.4$ & $12-18$ & 53 & $\leq 1$ & 54 & Present study \\
\hline
\end{tabular}

earlier reported composites, with a comparison given in Table 3 . Besides the EMI shielding applications, we firmly believe that the PANI composite, with optimum conductivity along with a high dielectric constant, may be the ultimate choice in many applications such as ESD, gas sensors, ${ }^{\mathbf{4 4}}$ thermoelectrics and energy applications ${ }^{45}$ etc.

\section{Conclusions}

A process for the synthesis of BST and EG-incorporated PANI composites using the micro emulsion method in large scale is demonstrated. The detailed micro-structural/structural characterizations as well as the characteristics of the core-shell structure have been examined by SEM, TEM, XRD, FTIR, Raman, UV and TGA. The size of the as-prepared BST particles was found to be $43 \mathrm{~nm}$ using XRD. Furthermore, our composites with different formulations are tuneable for microwave absorption and EMI shielding applications. The formulation containing a higher amount of EG (PBE112) could be useful for high-tech EMI shielding applications, while composites consisting of a higher amount of BST (PBE121) could be promising as a futuristic radar absorbing material.

\section{Acknowledgements}

The authors wish to thank Prof. R. C. Budhani, Director, NPL, for his keen interest in the work. The authors thank Dr N. Vijayan, K. N. Sood and Dr Vidyanand for recording XRD patterns and SEM and HRTEM micrographs, respectively.

\section{References}

1 Y. Huang, N. Li, Y. Ma, F. Du, F. Li, X. He, X. Lin, H. Gao and Y. Chen, Carbon, 2007, 45, 1614-1621.

2 N. Li, Y. Huang, F. Du, X. He, X. Lin, H. Gao, Y. Ma, F. Li, Y. Chen and P. C. Eklund, Nano Lett., 2006, 6, 1141-1145. 3 Y. Yang and M. C. Gupta, Nano Lett., 2005, 5, 2131-2134.
4 A. P. Singh, M. Mishra, P. Sambyal, B. K. Gupta, B. P. Singh, A. Chandra and S. K. Dhawan, J. Mater. Chem. A, 2013, 35813593.

5 M. Ashokkumar, T. N. Narayanan, B. K. Gupta, A. Leela Mohana Reddy, P. Avanish, S. K. Dhawan, C. Bangaru, D. S. Rawat, S. Talapatra and P. M. Ajayan, ACS Sustainable Chem. Eng., 2013, 619-626.

6 K.-Y. Park, J.-H. Han, S.-B. Lee, J.-B. Kim, J.-W. Yi and S.-K. Lee, Compos. Sci. Technol., 2009, 69, 1271-1278.

7 X. Huang, Z. Chen, L. Tong, M. Feng, Z. Pu and X. Liu, Mater. Lett., 2013, 111, 24-27.

8 A. P. Singh, A. Chandra and S. Dhawan, AIP Adv., 2011, 1, 022147.

9 M. Mishra, A. P. Singh and S. K. Dhawan, J. Alloys Compd., 2013, 557, 244-251.

10 A. Ohlan, K. Singh, A. Chandra, V. N. Singh and S. K. Dhawan, J. Appl. Phys., 2009, 106, 044305-044311.

11 M. Han and K. Zhao, J. Phys. Chem. C, 2008, 112, 1941219422.

12 G. Eda and M. Chhowalla, Nano Lett., 2009, 9(2), 814818.

13 X. Fu and D. D. L. Chung, Cem. Concr. Res., 1996, 26, 14671472.

14 H.-B. Zhang, W.-G. Zheng, Q. Yan, Y. Yang, J.-W. Wang, Z.-H. Lu, G.-Y. Ji and Z.-Z. Yu, Polymer, 2010, 51, 1191-1196.

15 C. Mao, X. Dong, T. Zeng, G. Wang and S. Chen, Mater. Res. Bull., 2007, 42, 1602-1610.

16 A. Yasmin, J.-J. Luo and I. M. Daniel, Compos. Sci. Technol., 2006, 66, 1182-1189.

17 A. P. Singh, M. Mishra, A. Chandra and S. K. Dhawan, Nanotechnology, 2011, 22, 9.

18 J. Q. Qi, Y. Wang, W. Ping Chen, L. Tu Li and H. Lai Wah Chan, J. Solid State Chem., 2005, 178, 279-284.

19 J. Zhu, S. Wei, L. Zhang, Y. Mao, J. Ryu, N. Haldolaarachchige, D. P. Younge and Z. Guo, J. Mater. Chem. A, 2011, 21, 3952-3959.

20 H. Zhang, C.-L. Mak, H. L.-W. Chan and X. Yao, Low Temperature Hybrid Processing Technology of Fine 
Electronic Ceramics, in Sintering Applications, ed. I. Burcu Ertuğ, Intech, 2013.

21 H. C. Pant, M. K. Patra, A. Verma, S. R. Vadera and N. Kumar, Acta Mater., 2006, 54, 3163-3169.

22 S.-H. Xie, B.-K. Zhu, X.-Z. Wei, Z.-K. Xu and Y.-Y. Xu, Composites, Part A, 2005, 36, 1152-1157.

23 A. Roy, A. Parveen, R. Deshpande, R. Bhat and A. Koppalkar, J. Nanopart. Res., 2013, 15, 1-11.

24 A. Parveen and A. S. Roy, J. Mater. Res., 2013, 1-8.

25 A. Parveen, A. R. Koppalkar and A. S. Roy, IEEE Sens. J., 2012, 12, 2817-2823.

26 X.-R. Zeng and T.-M. Ko, J. Polym. Sci., Part B: Polym. Phys., 1998, 35, 1993-2001.

27 S. Quillard, G. Louam, J. Buisson, M. Boyer, M. Lapkowski, A. Pron and S. Lefrant, Synth. Met., 1997, 84, 805-806.

28 T. K. Gupta, B. P. Singh, S. R. Dhakate, V. N. Singh and R. B. Mathur, J. Mater. Chem. A, 2013, 1, 9138-9149.

29 D. A. Tenne, A. M. Clark, A. R. James, K. Chen and X. X. Xi, Appl. Phys. Lett., 2001, 79, 3836-3839.

30 J. E. P. d. Silva, D. L. A. d. Faria, S. I. C. d. Torresi and M. L. A. Temperini, Macromolecules, 2000, 33, 3077-3083.

31 A. Shakoor, T. Z. Rizvi and A. Nawaz, J. Mater. Sci.: Mater. Electron., 2011, 22, 1076-1080.

32 A. P. Singh, P. Garg, F. Alam, K. Singh, R. B. Mathur, R. P. Tandon, A. Chandra and S. K. Dhawan, Carbon, 2012, 50, 3868-3875.

33 A. P. Singh, B. K. Gupta, M. Mishra, Govind, A. Chandra, R. B. Mathur and S. K. Dhawan, Carbon, 2013, 56, 86-96.

34 K. Chen, C. Xiang, L. Li, H. Qian, Q. Xiao and F. Xu, J. Mater. Chem., 2012, 22, 6449-6455.

35 B. Yuan, L. Yu, L. Sheng, K. An and X. Zhao, J. Phys. D: Appl. Phys., 2012, 45, 235108.
36 G.-S. Wang, X.-J. Zhang, Y.-Z. Wei, S. He, L. Guo and M.-S. Cao, J. Mater. Chem. A, 2013, 1, 7031-7036.

37 R. Che, L. M. Peng, X. Duan, Q. Chen and X. Liang, Adv. Mater., 2004, 16, 401-405.

38 P. Saini, M. Arora, G. Gupta, B. K. Gupta, V. N. Singh and V. Choudhary, Nanoscale, 2013, 5, 4330-4336.

39 M. Faisal and S. Khasim, Iran. Polym. J., 2013, 22, 473-480.

40 J. Liang, Y. Wang, Y. Huang, Y. Ma, Z. Liu, J. Cai, C. Zhang, H. Gao and Y. Chen, Carbon, 2009, 47, 922-925.

41 C. K. Das and A. Mandal, J. Mater. Sci. Res., 2012, 1, 45.

42 D. L. Leslie-Pelecky and R. D. Rieke, Chem. Mater., 1996, 8, 1770-1783.

43 Y.-J. Chen, P. Gao, R.-X. Wang, C.-L. Zhu, L.-J. Wang, M.-S. Cao and H.-B. Jin, J. Phys. Chem. C, 2009, 113, 1006110064.

44 M. K. Ram, Ö. Yavuz, V. Lahsangah and M. Aldissi, Sens. Actuators, B, 2005, 106, 750-757.

45 S. R. Gowda, A. Leela Mohana Reddy, X. Zhan and P. M. Ajayan, Nano Lett., 2011, 11, 3329-3333.

46 A. Ohlan, K. Singh, A. Chandra and S. Dhawan, Appl. Phys. Lett., 2008, 93, 053114.

47 T. K. Gupta, B. P. Singh, R. B. Mathur and S. R. Dhakate, Nanoscale, 2014, 6, 842-851.

48 K. Singh, A. Ohlan, V. H. Pham, R. Balasubramaniyan, S. Varshney, J. Jang, S. H. Hur, W. M. Choi, M. Kumar and S. Dhawan, Nanoscale, 2013, 2411-2420.

49 K. Wu, T. Ting, G. Wang, W. Ho and C. Shih, Polym. Degrad. Stab., 2008, 93, 483-488.

50 N. Gandhi, K. Singh, A. Ohlan, D. Singh and S. Dhawan, Compos. Sci. Technol., 2011, 71, 1754-1760.

51 Y. Bingqing, Y. Liming, S. Leimei, A. Kang and Z. Xinluo, J. Phys. D: Appl. Phys., 2012, 45, 235108. 